\title{
ORIGINAL
}

\section{INFLUENCIA DE LA DOBLE CARGA DE TRABAJO EN EL ESTADO DE SALUD PERCIBIDA Y UTILIZACIÓN DE SERVICIOS SANITARIOS DE LAS POBLACIONES INMIGRANTE Y AUTÓCTONA DE LA REGIÓN DE MURCIA}

\author{
Ana Belén Moreno López (1), Mónica Ballesta Ruiz (1), Diego Salmerón Martínez (1,2,3), Carmen \\ Navarro Sánchez $(1,2,3)$ y María José Tormo Díaz $(1,2,3)$.
}

(1) Servicio de Epidemiología. Consejería de Sanidad y Política Social, Murcia.

(2) CIBER Epidemiología y Salud Pública (CIBERESP), España.

(3) Departamento Ciencias Sociosanitarias. Universidad de Murcia, España.

Este trabajo recibó ayuda económicca de la Consejería de Sanidad de la Región de Murcia (BORM 08/11/2004), el Fondo para la Investigación Sanitaria del Instituto de Salud Carlos III (FIS ref. PI 052741) y del CIBER de Epidemiología y Salud Pública (CIBERESP) (Acción Específica nº PD08_004).

\section{RESUMEN}

Fundamentos: Las desigualdades de género en salud han sido ampliamente documentadas. El principal objetivo es evaluar si existen diferencias de género en salud percibida y utilización de servicios sanitarios, y su relación con la doble carga de trabajo, en una muestra representativa de población inmigrante y autóctona de la Región de Murcia (RM)

Métodos: Se utilizaron datos de la ENS 2006 y el Estudio Salud y Culturas, 1.303 inmigrantes y 1.303 españoles residentes en la RM. La combinación del trabajo reproductivo y remunerado se consideró "doble carga" (DC). Se estimó la razón de prevalencia (RP) de la percepción positiva de salud, morbilidad crónica, limitación de actividad, visitas al médico, hospitalización, visitas a urgencias y consumo de fármacos, en cada grupo de origen, mediante métodos de regresión. Se construyeron dos modelos, añadiendo el ajuste por DC al modelo ajustado por variables sociodemográficas. Se realizó análisis inter e intrasexo.

Resultados: Al ajustar por DC no se observaron cambios en las diferencias entre sexos [RP mujeres/hombres de percepción positiva salud: 0,70 (0,54-0,89) europeos Este; $0,87(0,79-0,95)$ autóctonos / morbilidad crónica: 1,44 (1,14-1,82) hispanoamericanos; $1,36(1,19-1,55)$ autóctonos / limitación actividad: $2,23(1,29-3,83)$ hispanoamericanos; $1,45(1,01-2,10)$ autóctonos / consulta médico: $1,93(1,50-2,48)$ hispanoamericanos; $1,74(1,06-2,86) \mathrm{ma}-$ rroquíes; 1,32 (1,09-1,59) autóctonos / hospitalización: 1,80 (1,02-3,17) hispanoamericanos], casi los mismos que sin ajustar. Las mujeres consumieron más fármacos que los hombres. Entre sexos, hombres $(1,19 ; 1,06-1,33)$ y mujeres $(1,18 ; 1,01-1,40)$ de la RM con DC compartida declararon mayor percepción positiva de salud que aquellos sin DC; hombres hispanoamericanos con DC sin ayuda: $0,67(0,47-0,94)$.

Conclusiones: Las mujeres presentan peores indicadores de salud y mayor uso de servicios sanitarios independientemente del origen. La doble carga no modifica las desigualdades de género en salud.

Palabras clave: Género. Desigualdades en salud. Servicios sanitarios. Inmigrantes.

\section{Correspondencia}

Ana Belén Moreno López.

Servicio de Epidemiología.

Consejería de Sanidad y Política Social de la Región de Murcia.

Ronda de Levante, 11

30008 Murcia (Spain)

anab.moreno2@carm.es.

\section{ABSTRACT}

Influence of Double Workload in Perceived Health and Health Services Utilization of Immigrants and Murcian Natives, Spain

Background: Gender inequalities in health have been largely documented. The main objective of this study is to assess whether there are gender differences in perceived health and health services utilization, and their relation with double workload in a representative sample of immigrants and Murcian natives.

Methods: We used data from the NHS 2006 and Health and Culture Study, 1,303 immigrants and 1,303 Spanish, both residents in the Region of Murcia. With the combination of reproductive work and paid work we built up the variable 'double workload' (DW). We estimated the prevalence ratio (PR) for positive self-perceived health, chronic morbidity, activity limitation, doctor's visits, hospitalization, emergency and drug use, by origin, using regression methods. Two models were constructed by adding double burden to the basic model adjusted by sociodemographic variables. Analyses were performed between and within sex.

Results: After adjusting for DW, no changes were seen in the differences by gender [RP women/men of positive perception health: $0.70(0.54-0.89)$ East European; 0.87 (0.79-0.95) autochthonous / chronic morbidity: 1.44 (1.14-1.82) Hispanic; $1.36(1.19-1.55)$ autochthonous / activity limitation: 2.23 (1.29-3.83) Hispanic; 1.45 (1.01-2.10) autochthonous / doctor's visits: 1.93 (1.50-2.48) Hispanic; 1.74 (1.06-2.86) Moroccan; 1.32 (1.09-1.59) autochthonous / hospitalization: 1.80 (1.02-3.17) Hispanic], almost the same than unadjusted. Women used more drugs than men. Within sexes, both autochthonous men $(1.19 ; 1.06-1.33)$ and women $(1.18 ; 1.01-1.40)$ with shared DW had more positive self-perceived health than those without DW. Hispanic men with DW without assistance: 0.67 (0.47-0.94).

Conclusions: Women have worse health indicators and greater use of health services regardless of origin. Consideration of the double workload does not explain gender inequalities in health.

Keywords: gender. Health inequalities. Health services. Immigrants. 


\section{INTRODUCCIÓN}

La observación repetida y documentada de que las mujeres perciben peor su salud y utilizan con más frecuencia los servicios sanitarios ha desarrollado un extenso campo de investigación sobre el impacto del género en la salud ${ }^{1-3}$, considerado este como una construcción social basada en las convenciones culturales, actitudes y relaciones entre hombres y mujeres ${ }^{4,5}$. La peor salud percibida por las mujeres se ha visto determinada por factores biológicos, psicológicos y sociales ${ }^{1,6}$. Adicionalmente, las mujeres pueden utilizar más los servicios sanitarios por diferencias en su rol social de cuidadora, sensibilidad a los síntomas, conocimientos en salud, disposición a comunicar los problemas de salud y una mayor adherencia al tratamiento ${ }^{7}$.

Diversos trabajos han puesto de manifiesto la existencia de desigualdades en salud relacionadas con el género ${ }^{6,8,9}$. El modelo social vigente, aunque en constante cambio, sigue asignando una división del trabajo por sexo ${ }^{9-12}$ que responsabiliza a los hombres de la manutención económica del hogar y a las mujeres del trabajo reproductivo, entendiendo por tal las tareas domésticas y el cuidado de los dependientes, especialmente los niños ${ }^{9,12,13}$. No obstante, es cada vez más frecuente que ambos sexos desempeñen paralelamente ambos roles, laboral y familiar, aunque en diferente medida, siendo mayoritario entre las mujeres. Esta doble carga de trabajo, traducida como un sobreesfuerzo personal, puede ser origen de alteraciones de la salud de diversa naturaleza.

La Encuesta Nacional de Salud 2006 (ENS 2006) concedió un gran protagonismo al enfoque de género en su diseño y análisis, incluyendo variables de trabajo reproductivo como el cuidado de menores de 15 años y las tareas domésticas ${ }^{14}$. Similarmente, el Estudio Salud y Culturas (Encuesta de salud en población inmigran- te de la Región de Murcia 2006-2007) ${ }^{15,16}$ incluyó dichas variables en el cuestionario pasado a la población inmigrante de mayor representación en su entorno.

Junto a las diferencias por género, también se han encontrado diferencias en salud en las personas que emigran por motivos económicos, percibiendo peor salud que la población que los recibe o permanece en el lugar de origen ${ }^{17-22}$. Durante la pasada década, la Región de Murcia (RM), al igual que otras regiones españolas, experimentó una afluencia masiva de población inmigrante que ha llegado a representar el $15 \%$ del total ${ }^{23}$. La nueva situación sociodemográfica obligó a estudiar las condiciones de salud y necesidades de asistencia sanitaria de los nuevos habitantes para racionalizar y adaptar el sistema sanitario, así como para garantizar la accesibilidad en condiciones de equidad. Aunque los resultados indican un uso de los servicios sanitarios similar al de los autóctonos, excepto en las urgencias, donde hay mayor frecuentación por parte de la población inmigrante, y en las hospitalizaciones -superiores en mujeres inmigrantes en edades reproductivas ${ }^{20,24,25}$, el estado de salud percibido es consistentemente peor en las mujeres de cualquiera de los lugares de origen de los inmigrantes y en las mujeres de la población autóctona ${ }^{15,16,26-28}$.

Dada la disponibilidad de múltiples datos sobre salud y género para un amplio rango de población de diferentes orígenes, el primer objetivo de este estudio fue describir la prevalencia del trabajo reproductivo, estado de salud declarado y utilización de servicios sanitarios de la población inmigrante y autóctona de la RM, según origen y sexo. El segundo consistió en evaluar si existían diferencias de género en salud percibida y utilización de servicios sanitarios y su relación con la doble carga de trabajo. 


\section{SUJETOS Y MÉTODOS}

Fuentes de información: Este trabajo se realizó a partir de los datos de dos estudios transversales llevados a cabo concurrentemente en 2006: Estudio Salud y Culturas y la ENS 2006.

El Estudio Salud y Culturas, cuya metodología detallada fue descrita previamente ${ }^{15}$, se llevó a cabo en una muestra representativa de la población inmigrante con Tarjeta Individual del Servicio Murciano de Salud, organismo responsable de la atención sanitaria pública de la RM. El método de muestreo fue estratificado, polietápico y por conglomerados. Se dividió a la población extranjera en 12 estratos que agrupaban los centros de salud de la Región. La selección de los centros de salud dentro de cada estrato se realizó con probabilidad proporcional al número de inmigrantes de cada centro en ese estrato. La selección de participantes en cada centro se llevó a cabo aleatoriamente hasta completar el número predeterminado proporcionalmente al origen, sexo y edad de cada estrato. El trabajo de campo fue llevado a cabo entre noviembre de 2006 y febrero de 2008 mediante entrevista personal por encuestadores entrenados. El cuestionario del Estudio Salud y Culturas se basó en el de la ENS $2006^{14}$, al que se añadieron preguntas que exploraban el proceso migratorio, siendo traducido al árabe, rumano, ruso, alemán y francés con doble traducción por personal nativo bilingüe. La tasa de respuesta global fue del $66,3 \%$, variando del $55,7 \%$ en marroquíes al $74,0 \%$ en europeos del Este ${ }^{16}$. Tras excluir a la población que no se encontraba en edad laboral, se entrevistó finalmente a 1.303 inmigrantes de 16 a 64 años, 672 $(52 \%)$ de origen hispanoamericano, 361 $(28 \%)$ de origen marroquí, $125(9 \%)$ de Europa Occidental y $145(11 \%)$ de Europa del Este. Tres participantes con doble nacionalidad fueron consideraron nativos.
Respecto a la ENS $2006^{14}$, elaborada por el Instituto Nacional de Estadística para el Ministerio de Sanidad, se utilizaron los microdatos de libre acceso, que constan de 29.478 entrevistas a personas de 16 y más años, de las que $1.491(5 \%)$ corresponden a residentes en la RM y $1.859(6 \%)$ a nacidos fuera de España sin nacionalidad española, siendo considerados en esta investigación como inmigrantes. La respuesta global obtenida fue del $96,1 \%$, del cual el $64,6 \%$ correspondió a hogares titulares y el 31,5\% restante a sustituciones ${ }^{29}$. Tras excluir a las personas de 65 y más años, se utilizó finalmente la información relativa a 19.822 nacidas en España o con doble nacionalidad $(\mathrm{n}=177)$, de las cuales 1.303 residían en la $\mathrm{RM}$, y la relativa a 1.788 personas nacidas fuera de España.

Muestra: Compuesta por los 1.303 (738 hombres y 565 mujeres) inmigrantes del Estudio Salud y Culturas junto a los 1.303 (440 hombres y 863 mujeres) autóctonos de la ENS 2006, residentes en la misma Región. Ambas bases de datos se unieron en una en la que cada persona tenía asignado su correspondiente peso muestral fuera de su fuente de origen. En el Estudio Salud y Culturas se ponderó, además, por el inverso de las tasas de respuesta.

Para el primer objetivo se utilizaron las variables de la población descrita, así como para el análisis intrasexo del segundo [(1.178 hombres (738 inmigrantes y 440 autóctonos) y 1.428 mujeres (565 inmigrantes y 863 autóctonas)].

El análisis intersexo del segundo objetivo requirió dos poblaciones diferentes. Para el estudio de la DC sobre los indicadores de salud y utilización de servicios sanitarios en población residente en la RM se usó la población definida anteriormente. Por otro lado, debido a que el Estudio Salud y Culturas no proporciona la información sobre el número de horas dedicadas al trabajo reproductivo, se usó la del total de participantes 
en la ENS 2006 menores de 65 años (19.822 autóctonos y 1.788 inmigrantes), a fin de poder valorar mejor la implicación individual en las tareas reproductivas.

Variables de estudio: Las variables analizadas proceden de las preguntas incluidas en el cuestionario de adultos de la ENS $2006^{30}$. En cuanto al nivel de salud, se exploró la percepción de la salud en el último año (En los últimos doce meses ¿diría que su estado de salud ha sido muy bueno, bueno, regular, malo, muy malo?), haber padecido alguna vez al menos uno de los 27 problemas crónicos de diversa naturaleza listados en la pregunta correspondiente (A continuación le voy a leer una lista con una serie de enfermedades o problemas de salud ¿padece o ha padecido alguna vez alguna de ellas?) y limitación de la actividad en las dos semanas previas a la entrevista (Durante las últimas dos semanas $i$ ha tenido que reducir o limitar sus actividades habituales al menos la mitad de un día por algunos dolores o síntomas?). Para este estudio se creó la variable dicotómica percepción positiva de salud a partir de las categorías del estado de salud percibido, considerándose que las personas entrevistadas que afirmaron tener un estado de salud bueno o muy bueno tenían percepción positiva y el resto no. Respecto a la utilización de los servicios sanitarios públicos, se estudió el número de visitas al médico de familia en el mes anterior a la encuesta (¿Cuántas veces ha consultado con un/a médico/a de familia en las últimas cuatro semanas por algún problema, molestia o enfermedad suya?), la utilización del servicio de urgencias (En estos últimos doce meses, ¿ha tenido que utilizar algún servicio de urgencias por algún problema o enfermedad?), los episodios de hospitalización en el año previo (Durante los últimos doce meses, ¿ha tenido que ingresar en un hospital como paciente al menos durante una noche?) y el consumo de fármacos con receta y sin receta en las 2 semanas anteriores a la entrevista como consumir al menos un medicamento (A continuación voy a leerle una lista de medicamentos, por favor dígame ¿cuál o cuáles de ellos ha consumido en las últimas dos semanas y cuáles le fueron recetados por el/la médico/a, independientemente de que se hayan consumido?).

Se consideraron contribución en las tareas domésticas las respuestas obtenidas a partir de la pregunta ¿Quién se ocupa principalmente de los trabajos de la casa como limpiar, cocinar, planchar, etc.? y contribución en el cuidado de los hijos a las obtenidas a partir de ¿Quién/es se ocupa/n habitualmente y de forma principal del cuidado de los niños menores de 15 años? Las posibilidades de respuesta para ambas preguntas eran: usted solo, usted compartiéndolo con su pareja, usted compartiéndolo con otra persona que no es su pareja, su pareja sola, otra persona de la casa que no es su pareja, una persona remunerada por ello, otra persona que no reside en el hogar, los servicios sociales, ninguna persona, otra situación. Se consideró que una persona estaba implicada cuando contestaba alguna de las tres primeras opciones. La variable implicación en el trabajo reproductivo (TR) se valoró en aquellas personas que contestaron positivamente a alguna de las dos preguntas anteriores, diferenciando si las realizaban sin ayuda o compartidas con otra persona. A partir de la respuesta a TR y solo para las personas con trabajo remunerado, considerado este como la situación laboral de empleado en la última semana, se creó la variable doble carga (DC) con 3 categorías ( $\sin$ ayuda: realizaba al menos una tarea reproductiva solo/a; compartida: realizaba una o ambas tareas reproductivas con ayuda de otra persona; negativa). El número de horas dedicadas al TR solo se recoge en la ENS 2006, por lo que su utilización se restringió al análisis de la percepción de salud de la muestra de esta encuesta. La variable se utilizó como duración media $( \pm \mathrm{DE})$ categorizada en tres grupos $(0,<3$ y $\geq 3$ horas /día) sobre la base de la distribución del total de la muestra.

Para ambas fuentes de datos se recogieron las variables sociodemográficas sexo, 
edad, nivel de estudios, clase social, convivencia con pareja, convivencia con menores de 15 años y origen.

Análisis estadístico: En relación al primer objetivo, para cada grupo de origen se calcularon las diferencias entre sexos de las características sociodemográficas, reproductivas (incluyendo la DC), el estado de salud y la utilización de servicios sanitarios. El contraste de proporciones se llevó a cabo mediante el test de la $\mathrm{chi}^{2}$.

Para el análisis intersexo del segundo objetivo se estimaron las razones de prevalencias (RP) entre sexos para cada grupo de origen, tomando como referencia a los hombres, mediante modelos lineales generalizados de Poisson. Para cada variable de estudio se construyeron dos modelos, uno básico (MB) ajustado por variables sociodemográficas (edad, nivel de estudios, clase social) y de estado de salud (percepción positiva de salud, problemas crónicos padecidos alguna vez y limitación de la actividad en las dos semanas previas a la entrevista) en el caso de las variables de utilización de servicios sanitarios. El segundo, modelo completo (MC) incorpora al primero la variable DC (trabajo doméstico y/o cuidado de los niños, además de trabajo remunerado). Para comparar las diferencias entre sexos en cada uno de los grupos con respecto a los nativos se construyeron modelos específicos para cada variable de estudio en los que se incluyó la interacción entre sexo y origen. Cuando el término de interacción entre el grupo estudiado y el nativo fue estadísticamente significativo $(\mathrm{p}<0,05)$, se concluyó que las diferencias entre sexos no eran iguales en ambos grupos.

Por otro lado, dentro del análisis intersexo se estimó el MB y el MC de la percepción positiva de salud, incorporando en el segundo modelo las horas dedicadas al TR en tres categorías $(0,<3$ y $\geq 3$ horas/día) para valorar su impacto adicional. Solo se utilizaron los datos de la ENS 2006 para la totalidad de la población española e inmigrante residente en España, por ser la única que proporciona dicha variable.

Respecto al análisis intrasexo, para evaluar el efecto diferencial de la DC sobre hombres y sobre mujeres se reprodujo el análisis de la percepción positiva de salud según la presencia o no de DC, añadiendo el ajuste por las características sociodemográficas. Este análisis se limitó a personas con empleo, requisito necesario para tener DC. Las personas sin DC se tomaron como grupo de referencia. Justificado por el diferente comportamiento de las mujeres marroquíes, la asociación en el total de mujeres inmigrantes se proporcionó incluyendo y excluyendo a este grupo.

Todos los análisis se realizaron con el programa estadístico STATA versión 10.0. Las estimaciones se presentaron como RP con sus respectivos intervalos de confianza del 95\% (IC95\%). Todos los modelos fueron ajustados por variables sociodemográficas. En todos los cálculos se utilizó el comando $s v y$, con pesos especificados como probability weights, fuera de la ENS 2006 y del Estudio Salud y Culturas.

\section{RESULTADOS}

Las características sociodemográficas, reproductivas, del estado de salud y utilización de servicios sanitarios según sexo y grupo de origen se muestran en la tabla 1 para la población inmigrante y autóctona residente en la RM. Las mujeres inmigrantes declararon estar empleadas $(79,1 \%)$ en menor proporción que los hombres $(94,7 \%)$, sobre todo en población marroquí (38,3\% vs $94,4 \%)$. En población murciana, declararon tener empleo el $44,6 \%$ de las mujeres y el $72,0 \%$ de los hombres. El desempeño del trabajo reproductivo fue más frecuente en las mujeres de cualquier origen. Las mujeres presentaron en mayor proporción DC sin ayuda (RM: 21,9\%; Europa Este: 64,9\%; Europa Occidental: 50,1\%; Hispanoaméri- 
Tabla 1

Distribución (\%) de las características sociodemográficas, reproductivas, de estado de salud y utilización de servicios sanitarios según origen y sexo de poblaciones inmigrante* y autóctona ${ }^{\dagger}$ residente en la Región de Murcia

\begin{tabular}{|c|c|c|c|c|c|c|c|c|c|c|c|c|c|c|c|c|c|c|}
\hline & \multicolumn{3}{|c|}{ Hispanoamérica } & \multicolumn{3}{|c|}{ Marruecos } & \multicolumn{3}{|c|}{ Europa Occidental } & \multicolumn{3}{|c|}{ Europa del Este } & \multicolumn{3}{|c|}{ Total inmigrantes } & \multicolumn{3}{|c|}{ Total nativos Murcia } \\
\hline & $\begin{array}{c}\text { Hombres } \\
n=351\end{array}$ & $\begin{array}{c}\text { Mujeres } \\
n=321\end{array}$ & $\mathrm{p}$ & $\begin{array}{c}\text { Hombres } \\
n=269\end{array}$ & $\begin{array}{c}\text { Mujeres } \\
n=92\end{array}$ & $\mathrm{p}$ & $\begin{array}{c}\text { Hombres } \\
n=49\end{array}$ & $\begin{array}{c}\text { Mujeres } \\
n=76\end{array}$ & $\mathrm{p}$ & $\begin{array}{c}\text { Hombres } \\
n=69\end{array}$ & $\begin{array}{c}\text { Mujeres } \\
n=76\end{array}$ & $\mathrm{p}$ & $\begin{array}{c}\text { Hombres } \\
\mathrm{n}=738\end{array}$ & $\begin{array}{c}\text { Mujeres } \\
n=565\end{array}$ & $\mathrm{p}$ & $\begin{array}{c}\text { Hombres } \\
n=440\end{array}$ & $\begin{array}{c}\text { Mujeres } \\
n=863\end{array}$ & $\mathrm{p}$ \\
\hline \multicolumn{19}{|c|}{ Sociodemográficas } \\
\hline Edad & & & 0,42 & & & 0,05 & & & 0,67 & & & 0,74 & & & 0,59 & & & 0,62 \\
\hline $16-24$ & 16,2 & 16,5 & & 15,8 & 24,9 & & 2,3 & 2,1 & & 14,7 & 14,2 & & 15,6 & 17,3 & & 20,0 & 16,7 & \\
\hline $25-34$ & 43,0 & 45,2 & & 44,6 & 37,3 & & 13,5 & 21,5 & & 47,0 & 41,3 & & 43,3 & 42,0 & & 22,7 & 22,4 & \\
\hline $35-44$ & 29,3 & 28,9 & & 27,5 & 28,9 & & 55,6 & 44,1 & & 29,2 & 29,8 & & 29,0 & 29,8 & & 25,0 & 25,2 & \\
\hline $45-64$ & 11,6 & 9,4 & & 12,1 & 9 & & 28,5 & 32,2 & & 9,0 & 14,6 & & 12,1 & 11,0 & & 32,3 & 35,7 & \\
\hline Nivel Estudios & & & 0,41 & & & 0,06 & & & 0,56 & & & 0,68 & & & 0,04 & & & 0,05 \\
\hline Elemental / Primarios & 50,9 & 47,1 & & 77,9 & 90,7 & & 11,0 & 16,9 & & 31,6 & 24,1 & & 62,4 & 52,7 & & 61,1 & 69,2 & \\
\hline Secundarios & 41,0 & 41,1 & & 15,4 & 4,7 & & 45,3 & 49,4 & & 51,0 & 60,1 & & 29,0 & 35,5 & & 20,3 & 18,6 & \\
\hline Universitarios & 8,1 & 11,8 & & 6,7 & 4,7 & & 43,6 & 33,7 & & 17,4 & 15,8 & & 8,5 & 11,8 & & 18,6 & 12,2 & \\
\hline Situación laboral última semana & & & 0,23 & & & 0,00 & & & 0,00 & & & 0,10 & & & 0,00 & & & 0,00 \\
\hline Empleado & 94,6 & 90,3 & & 94,4 & 38,3 & & 99,8 & 85,1 & & 96,5 & 89,9 & & 94,7 & 79,1 & & 72,0 & 44,6 & \\
\hline Clase Social & & & 0,06 & & & 0,23 & & & 0,68 & & & 0,10 & & & 0,51 & & & 0,54 \\
\hline I-III Direct/técnicos / manual cualif & 7,7 & 4,2 & & 4,5 & 0,5 & & 56,1 & 62,1 & & 5,2 & 8,3 & & 7,1 & 6,8 & & 46,1 & 42,5 & \\
\hline IV Manuales semi-cualificados & 52,8 & 41,3 & & 31,6 & 26,3 & & 42,5 & 34,4 & & 72,4 & 35,9 & & 43,1 & 38,7 & & 42,6 & 45,3 & \\
\hline V Manuales no cualificados & 39,5 & 54,5 & & 63,9 & 73,2 & & 1,4 & 3,5 & & 22,4 & 55,8 & & 49,8 & 54,6 & & 11,3 & 12,2 & \\
\hline Convive con pareja & 66,5 & 65,8 & 0,87 & 41,8 & 73,5 & 0,00 & 91,1 & 74,5 & 0,10 & 69,5 & 77,1 & 0,54 & 55,2 & 68,8 & 0,01 & 61,2 & 68,8 & 0,02 \\
\hline Convive con menores 15 años & 49,9 & 52,4 & 0,55 & 29 & 65,1 & 0,00 & 66,9 & 65,5 & 0,90 & 26,8 & 46,6 & 0,02 & 39,3 & 55,3 & 0,00 & 40,2 & 45,7 & 0,12 \\
\hline
\end{tabular}




\section{Tabla 1}

continuación

\begin{tabular}{|c|c|c|c|c|c|c|c|c|c|c|c|c|c|c|c|c|c|c|}
\hline & \multicolumn{3}{|c|}{ Hispanoamérica } & \multicolumn{3}{|c|}{ Marruecos } & \multicolumn{3}{|c|}{ Europa Occidental } & \multicolumn{3}{|c|}{ Europa del Este } & \multicolumn{3}{|c|}{ Total inmigrantes } & \multicolumn{3}{|c|}{ Total nativos Murcia } \\
\hline & $\begin{array}{c}\text { Hombres } \\
\mathrm{n}=351\end{array}$ & $\begin{array}{c}\text { Mujeres } \\
\mathrm{n}=321\end{array}$ & $\mathrm{p}$ & $\begin{array}{c}\text { Hombres } \\
\mathrm{n}=269\end{array}$ & $\begin{array}{c}\text { Mujeres } \\
\mathrm{n}=92\end{array}$ & $\mathrm{p}$ & $\begin{array}{c}\text { Hombres } \\
\mathrm{n}=49\end{array}$ & $\begin{array}{c}\text { Mujeres } \\
\mathrm{n}=76\end{array}$ & $\mathrm{p}$ & $\begin{array}{c}\text { Hombres } \\
\mathrm{n}=69\end{array}$ & $\begin{array}{c}\text { Mujeres } \\
\mathrm{n}=76\end{array}$ & $\mathrm{p}$ & $\begin{array}{c}\text { Hombres } \\
\mathrm{n}=738\end{array}$ & $\begin{array}{c}\text { Mujeres } \\
\mathrm{n}=565\end{array}$ & $\mathrm{p}$ & $\begin{array}{c}\text { Hombres } \\
\mathrm{n}=440\end{array}$ & $\begin{array}{c}\text { Mujeres } \\
\mathrm{n}=863\end{array}$ & $\mathrm{p}$ \\
\hline \multicolumn{19}{|c|}{ Reproductivas } \\
\hline Tareas domésticas & 57,6 & 88,8 & 0,00 & 67,6 & 91,6 & 0,00 & 62,8 & 81,9 & 0,04 & 71,9 & 97,5 & 0,00 & 63,1 & 89,7 & 0,00 & 29,1 & 78,4 & 0,00 \\
\hline Cuidado de menores de 15 años ${ }^{\ddagger}$ & 28,3 & 45,4 & 0,00 & 25,9 & 59,4 & 0,00 & 59,8 & 83,9 & 0,36 & 22,2 & 53,8 & 0,00 & 27,4 & 50,5 & 0,00 & 16,0 & 33,5 & 0,00 \\
\hline Trabajo reproductivo (TR) $)^{\|}$ & 22,5 & 42,9 & 0,00 & 16,0 & 58,0 & 0,00 & 35,2 & 48,8 & 0,33 & 13,2 & 46,5 & 0,00 & 19,3 & 46,7 & 0,00 & 8,6 & 31,5 & 0,00 \\
\hline Sin ayuda & 21.2 & 54.6 & & 39.2 & 13.8 & & 5.6 & 50.1 & & 25.6 & 64.9 & & 29.7 & 46.7 & & 4.6 & 21.9 & \\
\hline Compartida & 40.8 & 29.3 & & 36.5 & 22.3 & & 64.2 & 26.7 & & 52.1 & 22.5 & & 39.7 & 27.1 & & 22.9 & 13.4 & \\
\hline Negativa & 38.0 & 16.1 & & 24.3 & 63.9 & & 30.2 & 23.2 & & 22.3 & 12.5 & & 30.6 & 26.2 & & 72.5 & 64.7 & \\
\hline \multicolumn{19}{|c|}{ Estado de salud y utilización de servicios sanitarios } \\
\hline Percepción positiva de salud & 64,9 & 52,8 & 0,02 & 34,3 & 14,9 & 0,03 & 86,2 & 77,4 & 0,48 & 73,1 & 56,8 & 0,03 & 50,8 & 46,5 & 0,26 & 74,5 & 61,9 & 0,00 \\
\hline Al menos un problema crónico & 45,6 & 69,1 & 0,00 & 49,8 & 38,6 & 0,06 & 39,8 & 46,4 & 0,74 & 16,7 & 27,3 & 0,19 & 46,5 & 58,0 & 0,03 & 46,4 & 65,7 & 0,00 \\
\hline Consulta médico último mes & 22,6 & 42,9 & 0,00 & 25,5 & 51,9 & 0,00 & 28,4 & 50,5 & 0,16 & 12,0 & 21,8 & 0,17 & 23,7 & 43,5 & 0,00 & 28,3 & 42,0 & 0,00 \\
\hline Hospitalización último año & 5,0 & 9,7 & 0,00 & 7,3 & 22,5 & 0,00 & 9,0 & 13,1 & \begin{tabular}{|l|}
0,62 \\
\end{tabular} & 6,2 & 7,9 & 0,73 & 6,2 & 12,4 & 0,00 & 7,6 & 11,4 & 0,11 \\
\hline Urgencias último año & 38,6 & 46,8 & 0,07 & 58,4 & 70,3 & 0,15 & 29,7 & 36,6 & 0,49 & 20,2 & 19,1 & 0,77 & 47,3 & 48,9 & 0,61 & 31,2 & 31,8 & 0,87 \\
\hline Fármacos recetados últimas $2 \mathrm{sem}$ & 20,4 & 44,1 & 0,00 & 29,8 & 54,6 & 0,12 & 50,7 & 57,8 & 0,60 & 8,7 & 32,0 & 0,02 & 25,2 & 46,0 & 0,00 & 41,6 & 61,0 & 0,00 \\
\hline Consumo fármacos últimas $2 \mathrm{sem}$ & 35,4 & 55,1 & 0,00 & 35,1 & 59,8 & 0,08 & 50,9 & 64,1 & 0,31 & 28,5 & 55,2 & 0,19 & 35,3 & 56,5 & 0,00 & 52,3 & 68,6 & 0,00 \\
\hline
\end{tabular}

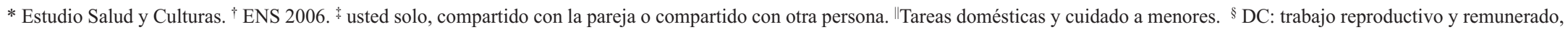
calculado exclusivamente para personas empleadas. 
ca: $54,6 \%$ ) y compartida los hombres (RM: 22,9\%; Europa Este: 52,1\%; Europa Occidental: 64,2\%; Hispanoamérica: 40,8\%), a excepción del origen marroquí (hombres: $39,2 \%$ sin ayuda; $36,5 \%$ compartida; mujeres: $13,8 \%$ sin ayuda; $22,3 \%$ compartida). La distribución detallada de las tareas domésticas y del cuidado de menores según sexo y origen puede observarse en las figuras 1 y 2 respectivamente. Las mujeres afirmaron realizar las tareas domésticas sin ayuda en mayor proporción que otras categorías en todos los orígenes (RM: 67,4\%; Europa Este: 72,4\%; Europa Occidental: 53,9\%; Marruecos: 52,2\%; Hispanoamérica: $54,8 \%$ ). Los hombres declararon con más frecuencia llevarlas a cabo de forma compartida con la pareja (RM: 19,3\%; Europa Este: 36,2\%; Europa Occidental: 59,2\%; Hispanoamérica: 33,9\%), a excepción de los marroquíes (40,9\% solos, 16,4\% junto a la pareja, $9,7 \%$ junto a otra persona). El cuidado de menores fue más frecuentemente realizado sin ayuda por las mujeres (RM: 54,8\%; Europa Este: 41,3\%; Europa
Occidental: 24,7\%; Marruecos: 57,1\%; Hispanoamérica: $50,0 \%$ ) y con ayuda de la pareja por los hombres (RM: 50,7\%; Europa Este: 42,5\%; Europa Occidental: 34,8\%; Marruecos: $80,9 \%$; Hispanoamérica: 48,9\%). Ningún hombre de Europa Occidental afirmó cuidarlos solo o con la ayuda de otra persona diferente a la pareja.

Una mayor proporción de hombres que de mujeres (RM: 74,5 vs 61,9\%; Europa Este: 73,1 vs 56,8\%; Europa Occidental: 86,2 vs $77,4 \%$; Marruecos: 34,3 vs $14,9 \%$; Hispanoamérica: 64,9 vs $52,8 \%$ ) informó percepción positiva de la salud en todos los orígenes (tabla 1). Los problemas crónicos de salud y la limitación de la actividad fueron más frecuentes en las mujeres hispanoamericanas $(69,1 \%$ vs $45,6 \%$ y $19,0 \%$ vs $9,4 \%$, respectivamente) y murcianas $(65,7 \%$ vs $46,4 \%$ y $18,6 \%$ vs $13,0 \%$, respectivamente). Las mujeres declararon mayor frecuentación de consulta médica (RM: 42,0 vs $28,3 \%$; Europa Este: 21,8 vs $12,0 \%$; Europa Occidental: 50,5 vs 28,4\%; Marruecos: 51,9 vs $25,5 \%$; Hispanoamérica: 42,9

Figura 1

Distribución de las tareas domésticas según sexo y origen

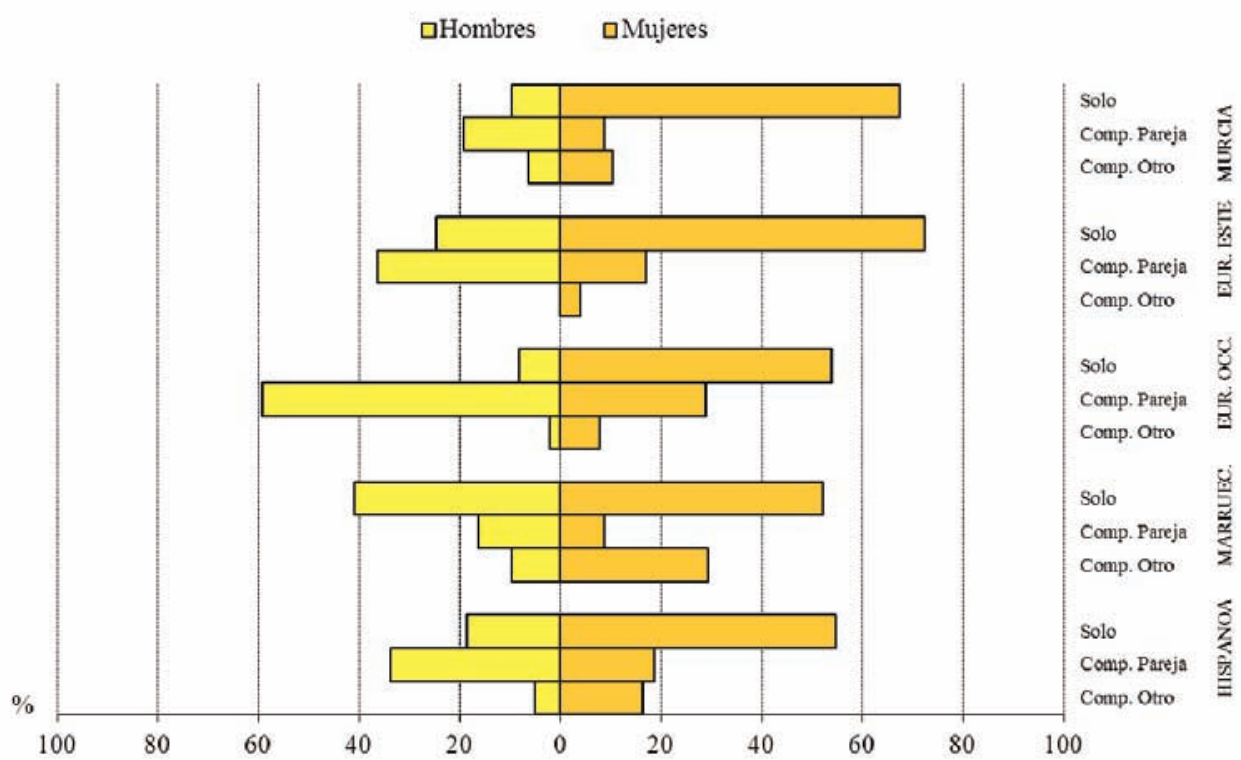




\section{Figura 2}

\section{Distribución del cuidado de menores de 15 años según sexo y origen}

口Hombres aMujeres

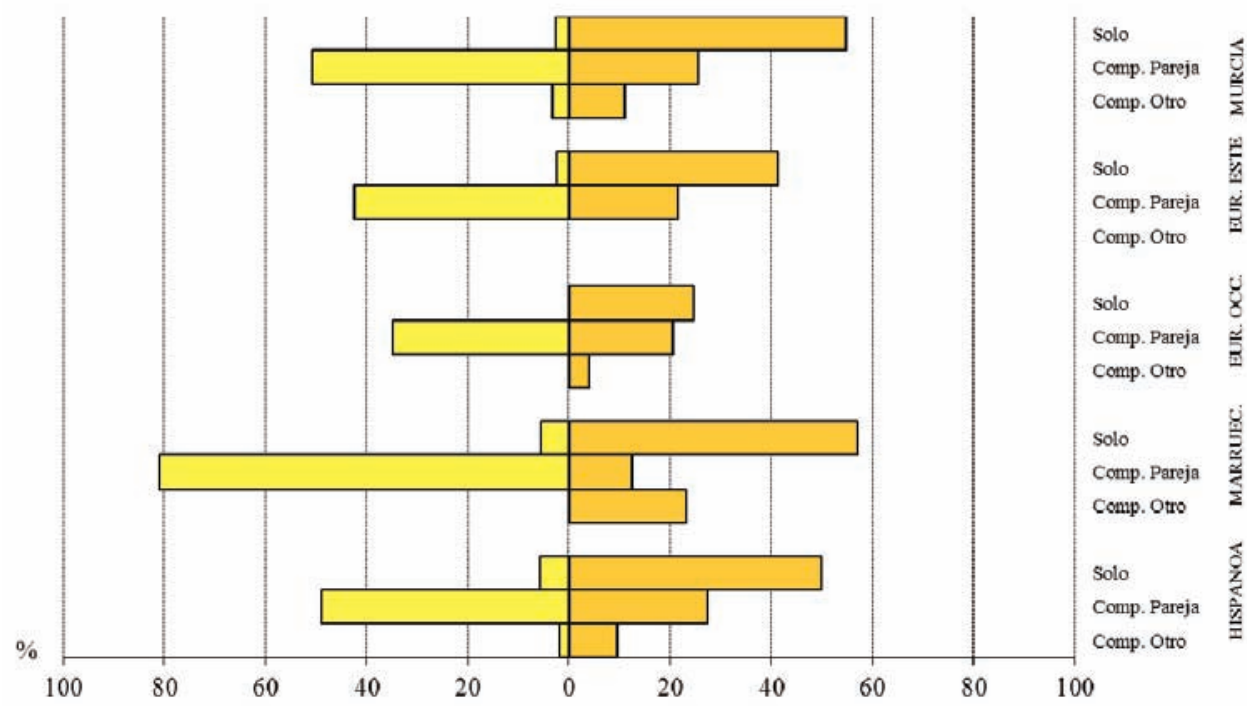

vs $22,6 \%$ ) y hospitalización (RM: 11,4 vs 7,6\%; Europa Este: 7,9 vs 6,2\%; Europa Occidental: 13,1 vs 9,0\%; Marruecos: 22,5 vs 7,3\%; Hispanoamérica: 9,7 vs $5,0 \%$ ) que los hombres en todos los orígenes. El consumo de fármacos, con y sin receta, también fue más frecuente entre las mujeres, sobre todo en las nativas de la RM (con receta: $61,0 \%$ - sin receta: $68,6 \%$ ).

En la tabla 2 se muestra el análisis intersexo de las variables de salud y utilización de servicios sanitarios, tomando como referencia a los hombres, según los diferentes grupos poblacionales residentes en la RM. La RP ajustada por DC de la percepción positiva de salud fue de 0,70 (IC95\%=0,540,89 ) en el colectivo de Europa del Este y $0,87(\mathrm{IC} 95 \%=0,79-0,95)$ en el colectivo murciano. Las mujeres inmigrantes presentaron al menos un problema crónico de salud un $26 \%$ más que los hombres $(1,26$; IC95\% $=1,04-1,53)$, un $44 \%$ en el caso de Hispanoamérica $(1,44$; IC95\%=1,14-1,82); en autóctonos se obtuvo una RP de 1,36 (IC95\%=1,19-1,55), significativamente diferente de la marroquí $(0,79$; IC95\%=1,53-1,17). La limitación de la actividad en las mujeres hispanoamericanas fue $2,23($ IC95\%=1,29-3,83) veces superior que en los hombres; en autóctonos se obtuvo una RP de 1,45 (IC95\%=1,01-2,10). Las visitas al médico fueron más frecuentes en mujeres autóctonas $(1,32$; IC $95 \%=1,09$ $1,59)$ y en inmigrantes $(1,86$; IC $95 \%=1,49$ $2,32)$; La RP de la población hispanoamericana $(1,93$; IC95\%=1,50-2,48) difirió significativamente con la RP de la autóctona. No se observaron diferencias estadísticamente significativas entre sexos en la asistencia a urgencias en ningún grupo. La hospitalización de mujeres de Hispanoamérica fue el $80 \%$ superior a la de hombres $(1,80$; IC95\%=1,02-3,17), 50\% superior en el total de inmigrantes $(1,50$; IC95\%=1,12-2,00). El consumo de fármacos fue superior en las mujeres de todos los orígenes, destacando las diferencias en el uso de fármacos con 


\section{Tabla 2}

Razón de prevalencia RP (IC 95\%) en estado de salud y utilización de servicios sanitarios de las mujeres frente a los hombres* según el origen de los inmigrantes y la población autóctona residente en la Región de Murcia

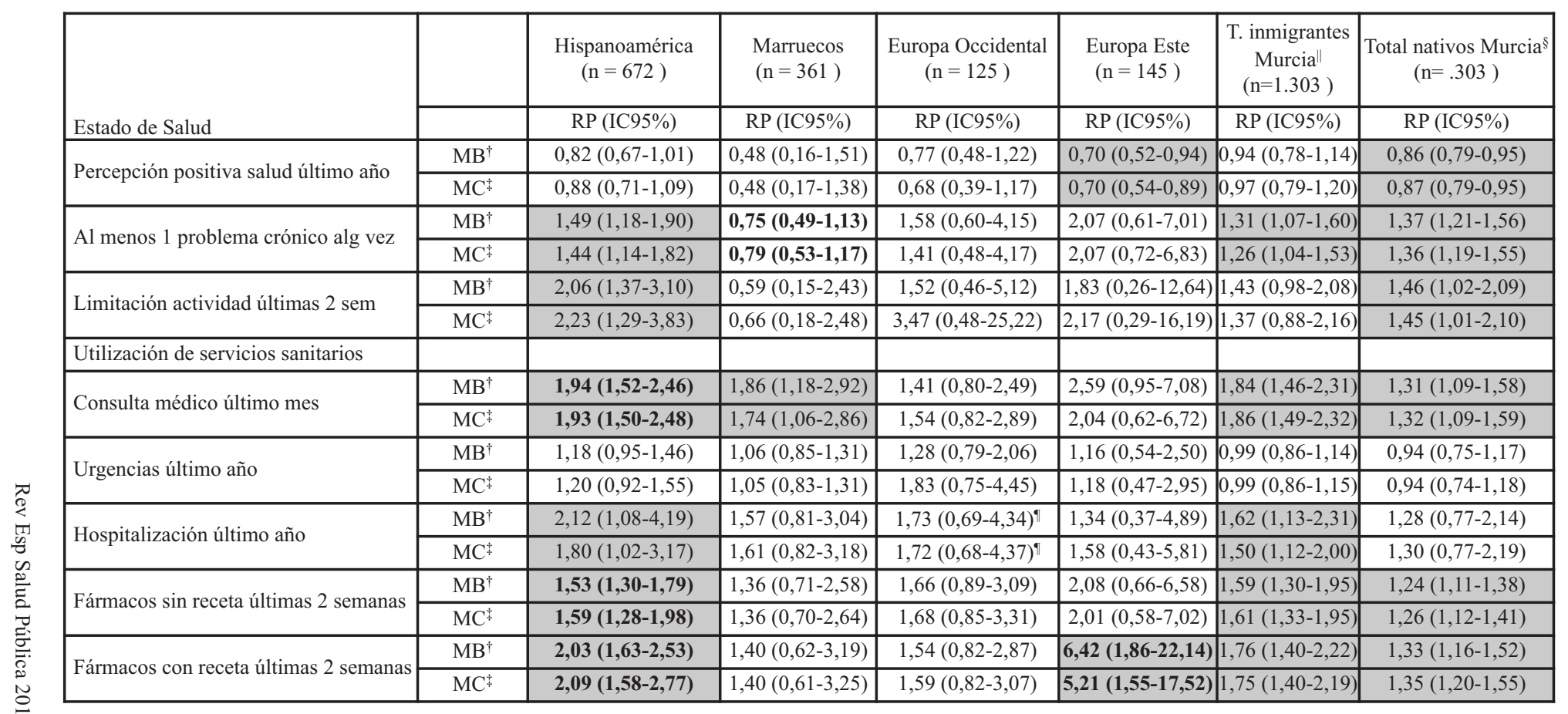

*Los hombres son la categoría de referencia. 'MB (Modelo básico): ajustado por variables sociodemográficas (edad, nivel estudios, clase social) y estado de salud para las variables de utilización servicios sanitarios. ${ }^{\star}$ MC (Modelo completo: MB ajustado por doble carga). " Estudio Salud y Culturas. ${ }^{\S}$ ENS $2006 .{ }^{\circledR}$ Para permitir la convergencia del modelo se ha agrupado la edad en 3 categorías (16-34, 35-44 y 45-64), nivel de estudios en 2 categorías (elemental, primario y secundarios y universitarios) y clase social en 2 categorías (I-III Directivos/técnicos/manual cualificados y IV-V Manuales semicualificados y no cualificados).Sombreados en gris se muestran los resultados estadísticamente significativos; en negrita, los grupos en los que el término interacción es significativo. 
Tabla 3

Razón de prevalencia de la percepción positiva de salud en mujeres frente a hombres* teniendo en cuenta el número de horas de trabajo reproductivo de las poblaciones inmigrante y autóctona residentes en España ${ }^{\dagger}$

\begin{tabular}{|c|c|c|}
\hline \multirow{2}{*}{ Modelo de ajuste } & \multicolumn{2}{|c|}{ RP (IC 95\%) } \\
\hline & $\begin{array}{l}\text { Total inmigrantes España } \\
\mathrm{n}=1.788\end{array}$ & $\begin{array}{c}\text { Total autóctonos España } \\
n=19.822\end{array}$ \\
\hline $\mathrm{MB}^{\dagger}$ & $0,85(0,78-0,92)$ & $0,90(0,88-0,92)$ \\
\hline $\mathrm{MB}+\mathrm{n}^{\circ}$ horas/día TR & $0,84(0,76-0,91)$ & $0,91(0,89-0,93)$ \\
\hline $\mathrm{MC}^{\|}$ & $0,86(0,79-0,93)$ & $0,90(0,88-0,92)$ \\
\hline $\mathrm{MC}+\mathrm{n}^{\mathrm{o}}$ horas/día $\mathrm{TR}^{\S}$ & $0,86(0,78-0,94)$ & $0,93(0,91-0,95)$ \\
\hline
\end{tabular}

RP (IC 95\%): Razón de prevalencia. *Los hombres son la categoría de referencia. ${ }^{\dagger}$ Modelo básico: ajustado por variables sociodemográficas (edad, nivel estudios, clase social). ${ }^{\ddagger}$ Modelo básico ajustado por $\mathrm{n}^{\circ}$ de horas dedicadas al trabajo reproductivo -TR- $(0 \mathrm{~h},<3 \mathrm{~h}, \geq 3 \mathrm{~h}) \|$ Modelo básico ajustado por doble carga (DC). ${ }^{\S}$ Modelo básico ajustado por DC y $\mathrm{n}^{\circ}$ de horas dedicadas al TR $(0 \mathrm{~h},<3 \mathrm{~h}, \geq 3 \mathrm{~h})$.

receta médica, sobre todo en Europa del Este (RP: 5,21; IC95\%=1,55-17,52) e Hispanoamérica (RP: 2,09; IC95\%=1,58-2,77). La razón entre sexos de ambos colectivos difirió significativamente de la autóctona $(1,35 ;$ IC95\%=1,20-1,55).

La tabla 3 presenta el análisis intersexo de la percepción positiva de salud sobre el total de población residente en España. La
RP en inmigrantes fue de 0,86 $(\mathrm{IC} 95 \%=0,79-0,93)$ en el MC y 0,86 $(\mathrm{IC} 95 \%=0,78-0,94)$ cuando se añadió al anterior el ajuste por el número de horas diarias dedicadas al TR. En autóctonos fue de $0,90($ I C $95 \%=0,88-0,92)$ y 0,93 $(\mathrm{IC} 95 \%=0,91-0,95)$, respectivamente.

En el análisis intrasexo (tabla 4), los hombres $(1,19$; IC95\%=1,06-1,33) y las mujeres

\section{Tabla 4}

\section{Razón de prevalencia de la percepción positiva de salud por sexo en personas con y sin doble carga} según origen de las poblaciones inmigrante* y autóctona ${ }^{\dagger}$ residentes en la Región de Murcia

\begin{tabular}{|l|c|c|c|c|c|c|}
\hline \multirow{2}{*}{\multicolumn{1}{|c|}{ Modelo de ajuste }} & \multicolumn{5}{|c|}{ RP (IC95\%) } \\
\cline { 2 - 7 } & Hispanoamérica & Marruecos & $\begin{array}{c}\text { Europa } \\
\text { Occidental }\end{array}$ & $\begin{array}{c}\text { Europa } \\
\text { Este }\end{array}$ & $\begin{array}{c}\text { Total inmigrantes } \\
\text { Murcia }\end{array}$ & $\begin{array}{c}\text { Total autóctonos } \\
\text { Murcia }\end{array}$ \\
\hline Mujeres: DC sin ayuda & $0,97(0,68-1,38)$ & $3,67(0,37-36,34)$ & $0,69(0,37-1,29)$ & $0,88(0,58-1,35)$ & $1,10(0,86-1,40)^{\ddagger}$ & $1,00(0,86-1,16)$ \\
\hline Mujeres: DC compartida & $0.93(0,59-1,44)$ & $3,39(0,32-35,62)$ & $\mathbf{0 , 3 1}(\mathbf{0 , 0 9 - 1 , 0 2 )}$ & $0,68(0,27-1,71)$ & $0,94(0,68-1,30)^{\ddagger}$ & $1,18(1,01-1,40)$ \\
\hline Hombres: DC sin ayuda & $\mathbf{0 , 6 7}(\mathbf{0 , 4 7 - 0 , 9 4 )}$ & $1,04(0,45-2,42)$ & $0,84(0,43-1,63)$ & $0,70(0,46-1,05)$ & $0,72(0,47-1,10)$ & $1,00(0,87-1,15)$ \\
\hline Hombres: DC compartida & $\mathbf{0 , 8 8} \mathbf{( 0 , 7 3 - 1 , 0 7 )}$ & $1,14(0,49-2,66)$ & $\mathbf{0 , 8 7}(\mathbf{0 , 6 9 - 1 , 1 1 )}$ & $0,78(0,41-1,46)$ & $0,90(0,65-1,26)$ & $1,19(1,06-1,33)$ \\
\hline
\end{tabular}

RP (IC 95\%): Razón de prevalencia.

*Estudio Salud y Culturas. ${ }^{\dagger}$ ENS 2006. ${ }^{\star}$ Excluyendo a las mujeres de Marruecos, la RP de mujeres con DC sin ayuda es 0,94 (0,73-1,23) y con DC compartida es $0,84(0,60-1,19)$. Modelos ajustados por variables sociodemográficas (edad, nivel estudios, clase social) y doble carga -DC- (personas sin DC son la categoría de referencia). Sombreados en gris se muestran los resultados estadísticamente significativos; en negrita, los grupos en los que el término interacción es significativo. 
$(1,18 ;$ IC $95 \%=1,01-1,40)$ de la RM con DC compartida declararon mayor percepción positiva de salud que aquellos sin DC. Los hombres hispanoamericanos con DC sin ayuda presentaron menor percepción positiva de salud que los que no tenían DC $(0,67$; IC95\%=0,47-0,94). Esta RP fue significativamente diferente a la equivalente en nativos. También se obtuvo un término de interacción significativo en el grupo de hombres de Hispanoamérica con DC compartida $(0,88$; IC95\% $\%=0,77-1,07)$ y en los grupos de Europa Occidental con DC compartida (hombres: 0,87; IC95\%=0,69-1,11 / mujeres: 0,31; IC95\% $=0,09-1,02)$.

\section{DISCUSIÓN}

La realización del trabajo reproductivo, tanto las tareas domésticas como el cuidado de menores, recae mayoritariamente sobre las mujeres de todos los orígenes. Estas diferencias entre sexos se intensifican cuando se observa la práctica de dichas actividades con mayor detalle. Así, las mujeres llevan a cabo el trabajo reproductivo sin ninguna ayuda en mayor proporción que los hombres, con diferencias variables pero de gran magnitud en todos los grupos, a excepción de las tareas domésticas en los marroquíes donde se reparten más equitativamente. Las desigualdades mencionadas son especialmente manifiestas en el grupo de Murcia y Europa Occidental para las tareas domésticas y en todos los grupos para el cuidado de menores. Los hombres realizan el trabajo reproductivo compartido con su pareja fundamentalmente. Esta tendencia se invierte cuando la colaboración se lleva a cabo con otra persona que no es la pareja, llegando incluso a no haber ningún hombre en esta situación en los colectivos marroquí, europeos occidentales y del Este. Diversas investigaciones del ámbito nacional han mostrado diferencias en las horas de dedicación a dichas tareas: los hombres dedican más tiempo al trabajo remunerado y menos a las tareas reproductivas ${ }^{12,31}$.

En población autóctona, las mujeres pre- sentan peor nivel de salud percibida, mayor frecuentación al médico de familia y un consumo más elevado de medicamentos que los hombres. Las principales desigualdades de género en salud y uso de servicios sanitarios en población inmigrante se encuentran en el grupo hispanoamericano, destacando las hospitalizaciones, visitas al médico de familia y el consumo de fármacos. A pesar de ser este el colectivo con mayor similitud al murciano en la relación entre sexos, existen diferencias entre ambos en los dos últimos recursos mencionados. Las mujeres marroquíes tienen mayor frecuentación al médico general que los hombres marroquíes. Las europeas del Este consumen fármacos recetados en una proporción muy superior a los hombres del mismo grupo. Esta diferencia es significativamente mayor al que presenta la población autóctona. Las relaciones entre hombres y mujeres de Europa Occidental parecen estar en la línea del resto de grupos, no obstante, la imprecisión de los resultados impide extraer conclusiones. El hecho de que los extranjeros de países occidentales, con mayor tradición igualitaria, no presenten diferencias entre sexos hace pensar que otros factores de tipo sociocultural no medidos, como la educación recibida, puedan reducir las desigualdades halladas en otros grupos.

Los hallazgos de este estudio concuerdan con otros publicados en el ámbito nacional e internacional. Así, la desventaja en el nivel de salud de las mujeres se reitera en múltiples investigaciones en población inmigrante y autóctona ${ }^{3,17,18,32}$. En cuanto al consumo de fármacos, una investigación realizada a partir de los datos de la ENS 2006 asoció más automedicación a los grupos de Europa Central y del Este ${ }^{33}$. En nuestro estudio se observó también una proporción superior entre los individuos de Europa del Este, con distribución por sexos similar.

La DC no modifica significativamente las desigualdades entre hombres y muje$\operatorname{res}^{34}$ en ninguna variable evaluada. El aná- 
lisis realizado sobre la salud percibida en el total de población inmigrante y autóctona residente en España, incorporando el número de horas dedicado al trabajo reproductivo, corrobora dicha observación. Aún con la enorme potencia de utilizar toda la muestra de la ENS 2006, la diferente percepción de salud entre sexos no varía al introducir la DC y el número de horas de tareas reproductivas.

En el análisis intrasexo de la percepción positiva de la salud no se observa un patrón por sexo. Por el contrario, se desprende un patrón por origen en el que hombres y mujeres de la RM y Marruecos tienen mejor percepción de su salud cuando combinan trabajo remunerado y tareas reproductivas, sobre todo cuando se realizan de forma compartida con otra persona, a la inversa de lo que ocurre con el resto de colectivos.

Este estudio no llega a explicar finalmente la razón por la cual las mujeres, independientemente de su origen, perciben peor su salud y utilizan más los servicios sanitarios que los hombres. Aparte de las explicaciones biomédicas que indican la necesidad de ajustar por el número de patologías y no solo por su presencia ${ }^{35}$, las ciencias sociales centran la justificación de este hecho en la diferente exposición y vulnerabilidad a los determinantes sociales de la salud ${ }^{36}$, siendo los más relevantes para las mujeres los factores estructurales (socioeconómicos, edad, soporte social, estructura familiar) y psicosociales (eventos críticos, estresores crónicos, recursos psicológicos), y los conductuales o estilos de vida para los hombres ${ }^{37-40}$.

En relación a los múltiples roles, diversos investigadores han concluido que, a pesar de que pueden ocasionar limitación de tiempo y energía ${ }^{9,12,34}$, en general no tienen un efecto negativo sobre la salud, ya que se relacionan con una mayor autoestima, prestigio, apoyo social y privile- $\operatorname{gios}^{41,42}$. No obstante, el efecto que producen depende en mayor medida de la calidad de los roles y la satisfacción asociada, la cual varía en función de múltiples factores como el momento vital, el contexto socioeconómico, tipo de trabajo y jornada laboral $^{41,43,44}$.

En la interpretación de estos resultados se han de considerar las posibles limitaciones del estudio, como su naturaleza transversal, por lo que se han restringido las conclusiones a lo observado sin imputar temporalidad. El efecto de la relativa baja tasa de respuesta obtenida en la población marroquí y en los individuos más jóvenes se intentó minimizar empleando el inverso de las tasas de respuesta. Para reducir el efecto del inmigrante sano se ajustó por los factores socioeconómicos y sanitarios ${ }^{45}$. Aunque los análisis se han limitado a personas con trabajo remunerado, no se ha considerado el número de horas empleadas, lo cual se ha relacionado con el estado de salud ${ }^{31}$. La incorporación del número de horas dedicadas al trabajo reproductivo contrarresta el efecto de no incluir el número de convivientes en el hogar. El método de regresión de Poisson utilizado es robusto, aunque en algunos grupos puede haber indefinición debido al relativamente escaso tamaño muestral.

La principal fortaleza de esta investigación es la incorporación de la perspectiva de género al estudio del estado de salud y utilización de servicios sanitarios a partir de una muestra representativa de población ${ }^{18,46}$. Además, se realiza el estudio individualizado de los diferentes grupos de población inmigrante, tal y como se recomienda ${ }^{20}$.

Concluyendo, las mujeres se ocupan mayoritariamente de las tareas reproductivas. A su vez, presentan menor percepción positiva de la salud, mayor morbilidad crónica y limitación de la actividad, así como una utilización superior de servi- 
cios sanitarios que los hombres. La doble carga de trabajo no modifica significativamente las desigualdades en salud entre hombres y mujeres en ningún grupo de población estudiado. Es necesario incluir en los modelos otros factores sociales que contribuyan a esclarecer las diferencias de género en salud.

\section{AGRADECIMIENTOS}

Reconocemos la ayuda facilitada por instituciones, profesionales e inmigrantes que nos concedieron parte de su escaso tiempo.

\section{BIBLIOGRAFÍA}

1. World Health Organization. Gender, Health and Aging. Geneva: World Health Organization; 2003. Disponible en: http://www.who.int/gender/documents/en/Gender_Ageing.pdf

2. Borrell C, García-Calvente MM, Martí-Boscà JV. Informe SESPAS 2004. La salud pública desde la perspectiva de género y clase social. Gac Sanit. 2004;18(Supl 1):2-6.

3. Bambra C, Pope D, Swami V, Stanistreet D, Roskam A, Kunst A, et al. Gender, health inequalities and welfare state regimes: a cross-national study of 13 European countries. J Epidemiol Community Health. 2009;63(1):38-44.

4. World Health Organization. Integrating gender perspective in the work of WHO. Geneva: World Health Organization; 2002. Disponible en: http://www.who.int/gender/documents/engpolicy.pd f

5. Krieger N. A glossary for social epidemiology. J Epidemiol Community Health. 2001;55:693-700.

6. Observatorio de Salud de la Mujer. Dirección General de la Agencia de Calidad del Ministerio de Sanidad y Consumo. Informe salud y género 2005. Madrid: Ministerio de Sanidad y Consumo; 2007. Disponible en http://www.msssi.gob.es/organizacion/sns/planCalidadSNS/docs/informe2005SaludGenero.pdf

7. Green CA, Pope CR. Gender, psychosocial factors and the use of medical services: a longitudinal analysis. Soc Sci Med. 1999;48(10):1.363-72.
8. Artazcoz L, Borrell C, Benach J, Cortes I, Rohlfs I. Women, family demands and health: the importance of employment status and socio-economic position. Soc Sci Med 2004;59(2):263-74.

9. Borrell C, Muntaner C, Benach J, Artazcoz L. Social class and self-reported health status among men and women: what is the role of work organisation, household material standards and household labour? Soc Sci Med. 2004;58(10):1869-87.

10.Borrell C, Artazco L. Las desigualdades de género en salud: retos para el futuro. Rev Esp Salud Pública. 2008;82(3):245-9.

11. Larrañaga I, Arregib B, Arpalb J. Informe SESPAS 2004. El trabajo reproductivo o doméstico. Gac Sanit. 2004;18(Supl 1):31-7.

12. Artazcoz L, Escriba-Aguir V, Cortes I. Género, trabajos y salud en España [Gender, paid work, domestic chores and health in Spain]. Gac Sanit. 2004; 18 (Suppl 2): $24-35$..

13. Borrell C, Muntaner C, Sola J, Artazcoz L, Puigpinos R, Benach J, et al. Immigration and self-reported health status by social class and gender: the importance of material deprivation, work organisation and household labour. J Epidemiol Community Health. 2008;62(5):e7

14. Ministerio de Sanidad y Consumo. Encuesta Nacional de Salud 2006. Metodología detallada. Madrid: Ministerio de Sanidad y Consumo; 2007. Disponible en: http://www.msssi.gob.es/estadEstudios/estadisticas/encu estaNacional/encuestaNac2006/metodENS2006.pdf

15. Colorado-Yohar S, Tormo MJ, Salmeron D, Dios S, Ballesta M, Navarro C. Violence reported by the immigrant population is high as compared with the native population in southeast Spain. J Interpers Violence. 2012;27(16):3322-40.

16. Tormo MJ, Ballesta M, Salmerón D, Navarro C. Estado de salud percibido en la población inmigrante en la Región de Murcia. Comparación con la población nativa a partir de la Encuesta Nacional de Salud 2006. Estudio Salud y Culturas. Bol Epidemiol Murcia 2010;30:105-8.

17. Cooper H. Investigating socio-economic explanations for gender and ethnic inequalities in health. Soc Sci Med. 2002;54(5):693-706.

18. Gerritsen AA, Deville WL. Gender differences in health and health care utilisation in various ethnic groups in the Netherlands: a cross-sectional study. BMC Public Health. 2009;9:109. 
19. Quan H, Fong A, De CC, Wang J, Musto R, Noseworthy TW, et al. Variation in health services utilization among ethnic populations. CMAJ. 2006;174(6):787-91.

20. Regidor E, Sanz B, Pascual C, Lostao L, Sanchez E, az Olalla JM. Health services utilization by the immigrant population in Spain. Gac Sanit. 2009;23(Suppl $1): 4-11$.

21. Reijneveld SA. Reported health, lifestyles, and use of health care of first generation immigrants in The Netherlands: do socioeconomic factors explain their adverse position? J Epidemiol Community Health. 1998;52(5):298-304.

22.Wiking E, Johansson SE, Sundquist J. Ethnicity, acculturation, and self reported health. A population based study among immigrants from Poland, Turkey, and Iran in Sweden. J Epidemiol Community Health. 2004;58(7):574-82.

23. Centro Regional de Estadística de Murcia. Anuario Estadístico de la Región de Murcia 2006. Murcia: Centro Regional de Estadística de Murcia; 2007. Disponible en: http:/www.carm.es/econet/anuario/a2007/anuario_tom oI.html

24. Hernando L, Palomar J, Márquez M, Monteagudo O. Impact of inmigration on hospital care: utilization, casemix, and economic effects. Gac Sanit. 2009;23:208-15.

25. Lopez NA, Ramos Parreno JM. [Health services utilization by the immigrant and native-born populations in the autonomous region of Murcia (Spain)]. Gac Sanit. 2009;23(Suppl 1):12-8.

26. del Amo J, Jarrin I, Garcia-Fulgueiras A, IbanezRojo V, Alvarez D, Rodriguez-Arenas MA, et al. Mental health in Ecuadorian migrants from a population-based survey: the importance of social determinants and gender roles. Soc Psychiatry Psychiatr Epidemiol. 2011;46(11):1143-52.

27. Llacer A, del Amo JD, Garcia-Fulgueiras A, IbanezRojo V, Garcia-Pino R, Jarrin I, et al. Discrimination and mental health in Ecuadorian immigrants in Spain. J Epidemiol Community Health. 2009;63(9):766-72.

28. Rodriguez AE, Lanborena EN, Senhaji M, Pereda RC. Sociodemographic variables and lifestyle as predictors of self-perceived health in immigrants in the Basque Country [Spain]. Gac Sanit. 2008;22(5):404-12.

29. Instituto Nacional de Estadística. Evaluación de la falta de respuesta en la Encuesta Nacional de Salud 2006. Madrid: Instituto Nacional de Estadística; 2007 Disponible en: http://www.ine.es/daco/daco42/sanitarias/evaluacion06.pdf
30. Ministerio de Sanidad y Consumo. Encuesta Nacional de Salud 2006. Cuestionario de adultos (Personas de 16 y más años). Madrid: Ministerio de Sanidad y Consumo; 2007. Disponible en: http://www.msssi.gob.es/estadEstudios/estadisticas/encuestaNacional/encuestaNac2006/EN S_06_Adultos_definitivo.pdf

31. Artazcoz L, Artieda L, Borrell C, Cortés I, Benach J, García V. Combining job and family demands and being healthy. Eur J Public Health 2004;14:43-8.

32. Esteban-Vasallo MD, Dominguez-Berjon MF, AstrayMochales J, Genova-Maleras R, Perez-Sania A, SanchezPerruca L, et al. Prevalence of diagnosed chronic disorders in the immigrant and native population. Gac Sanit. 2009;23(6):548-52.

33. Jimenez RD, Hernandez QC. Differences in selfmedication in the adult population in Spain according to country of origin. Gac Sanit. 2010;24(2):116-8.

34. Artazcoz L, Borrell C, Benach J. Gender inequalities in health among workers: the relation with family demands. J Epidemiol Community Health. 2001; 55(9): 639-47.

35. Malmusi D, Artazcoz L, Benach J, Borrell C. Perception or real illness? How chronic conditions contribute to gender inequalities in self-rated health. Eur J Public Health. 2011:16.

36. Verbrugge LM. Gender and health: an update on hypotheses and evidence. J Health Soc Behav. 1985; 26 (3):156-82.

37. Arber S, Lahelma E. Inequalities in women's and men's ill-health: Britain and Finland compared. Soc Sci Med. 1993; 37 (8): 1055-68.

38. Denton M, Prus S, Walters V. Gender differences in health: a Canadian study of the psychosocial, structural and behavioural determinants of health. Soc Sci Med. 2004; 58 (12): 2585-600.

39. Vives A, Vanroelen C, Amable M, Ferrer M, Moncada $\mathrm{S}$, Llorens C, et al. Employment precariousness in Spain: prevalence, social distribution, and population-attributable risk percent of poor mental health. Int J Health Serv. 2011;41(4):625-46.

40. Menéndez M, Benach J, Muntaner C, Amable M, O'Campo P. Is precarious employment more damaging to women's health than men's? Soc Sci Med. 2007; 64(4): 776-81.

41. Barnett RC, Hyde JS. Women, men, work, and family: An Expansionist Theory. American Psychologist. 2001; 56(10): 781-96. 
42. Matud Aznar MP. Género y salud. Suma Psicol. 2008;15(1):75-94

43. Macintyre S, Hunt K, Sweeting H. Gender differences in health: Are things really as simple they seem? Soc Sci Med. 1996; 42 (4): 617-24.

44. Klumb PL, Lampert T. Women, work and well-being 1950-2000: a review and methodological critique. Soc Sci Med. 2004; 58(6):1007-24.)

45. Choi BC. Definition, sources, magnitude, effect modifiers, and strategies of reduction of the healthy worker effect. J Occup Med. 1992;34(10):979-88.

46. Llacer A, Zunzunegui MV, del Amo J, Mazarrasa L, Bolumar F. The contribution of a gender perspective to the understanding of migrants' health. J Epidemiol Community Health. 2007;61(Supp1 2):ii4-10. 